\title{
Erratum to: On computing ellipsoidal harmonics using Jekeli's renormalization
}

\author{
Josef Sebera · Johannes Bouman · Wolfgang Bosch
}

Published online: 10 April 2012

(c) Springer-Verlag 2012

\section{Erratum to: J Geod}

\section{DOI 10.1007/s00190-012-0549-4}

An error in the equations had occurred in the original version of the online published article. Corrections are indicated by exclamation marks.

$* \bar{S}_{n, m}^{0}$ recurrence-referring to Eq. (20)

${ }^{*} \bar{S}_{n, m}^{0}={ }^{*} \bar{S}_{n, m}^{0}\left(\frac{u}{E}\right)=\beta_{n, m} \sum_{k=0}^{\infty} \alpha_{n, m, k}$

$\alpha_{n, m, k}=\frac{-(n \stackrel{!}{-} m+2 k-1)(n-m+2 k)}{2 k(2 n+2 k+1)}\left(\frac{E}{u}\right)^{2} \alpha_{n, m, k-1}$

$\alpha_{n, m, 0}=1$

$\beta_{n, m}=\underbrace{\frac{u}{\sqrt{u^{2}+E^{2}}}}_{!} \beta_{n, m-1}$

The online version of the original article can be found under doi:10.1007/s00190-012-0549-4.

J. Sebera

Faculty of Civil Engineering, CTU in Prague, Thákurova 7,

16629 Praha 6, Czech Republic

\section{J. Sebera ( $\square)$}

Astronomical Institute of the Academy of Sciences of the Czech

Republic, Ondřejov, Czech Republic

e-mail: josef.sebera@fsv.cvut.cz

\section{J. Bouman · W. Bosch}

Deutsches Geodätisches Forschungsinstitut,

Alfons-Goppel-Strasse 11, 80539 München, Germany

e-mail: bouman@dgfi.badw.de

W. Bosch

e-mail: bosch@dgfi.badw.de $\beta_{n, 0}=\frac{R}{u} \beta_{n-1,0}$

$\beta_{0,0}=\frac{R}{u}$

$* \bar{S}_{n, m}^{1}$ recurrence-referring to Eq. (21)

$* \bar{S}_{n, m}^{1}=\frac{\partial \bar{S}_{n, m}^{0} *}{\partial u}=\frac{\partial \beta_{n, m}}{\partial u} \sum_{k=0}^{\infty} \alpha_{n, m, k}+\beta_{n, m} \sum_{k=0}^{\infty} \frac{\partial \alpha_{n, m, k}}{\partial u}$

$\frac{\partial}{\partial u} \alpha_{n, m, k}=\frac{-(n-m+2 k-1)(n-m+2 k)}{2 k(2 n+2 k+1)}$

$\times\left(-2 \frac{E^{2}}{u^{3}} \alpha_{n, m, k-1}+\frac{E^{2}}{u^{2}} \frac{\partial \alpha_{n, m, k-1}}{\partial u}\right)$

$\frac{\partial \alpha_{n, m, 0}}{\partial u}=0$

$\frac{\partial \beta_{n, m}}{\partial u}=\underbrace{\frac{E^{2}}{\left(u^{2}+E^{2}\right)^{\frac{3}{2}}}}_{!} \beta_{n, m-1}+\underbrace{\frac{u}{\sqrt{u^{2}+E^{2}}}}_{!} \frac{\partial \beta_{n, m-1}}{\partial u}$

$\frac{\partial \beta_{n, 0}}{\partial u}=-\frac{R}{u^{2}} \beta_{n-1,0}+\frac{R}{u} \frac{\partial \beta_{n-1,0}}{\partial u}$

$\frac{\partial \beta_{0,0}}{\partial u}=-\frac{R}{u^{2}}$

$* \bar{S}_{n, m}^{2}$ recurrence: referring to Eq. (22)

$$
\begin{aligned}
* \bar{S}_{n, m}^{2}= & \frac{\partial^{2 *} \bar{S}_{n, m}^{0}}{\partial u^{2}}=\frac{\partial^{2} \beta_{n, m}}{\partial u^{2}} \sum_{k=0}^{\infty} \alpha_{n, m, k} \\
& +2 \frac{\partial \beta_{n, m}}{\partial u} \sum_{k=0}^{\infty} \frac{\partial \alpha_{n, m, k}}{\partial u}+\beta_{n, m} \sum_{k=0}^{\infty} \frac{\partial^{2} \alpha_{n, m, k}}{\partial u^{2}}
\end{aligned}
$$




$$
\begin{aligned}
& \frac{\partial^{2} \alpha_{n, m, k}}{\partial u^{2}}=\frac{-(n-m+2 k-1)(n-m+2 k)}{2 k(2 n+2 k+1)} \\
& \times\left(6 \frac{E^{2}}{u^{4}} \alpha_{n, m, k-1}-4 \frac{E^{2}}{u^{3}} \frac{\partial \alpha_{n, m, k-1}}{\partial u}\right. \\
& \left.+\frac{E^{2}}{u^{2}} \frac{\partial^{2} \alpha_{n, m, k-1}}{\partial u^{2}}\right) \\
& \frac{\partial^{2} \alpha_{n, m, 0}}{\partial u^{2}}=0 \\
& \frac{\partial^{2} \beta_{n, m}}{\partial u^{2}}=\underbrace{-\frac{3 u E^{2}}{\left(u^{2}+E^{2}\right)^{\frac{5}{2}}}}_{!} \beta_{n, m-1}+\underbrace{2 \frac{E^{2}}{\left(u^{2}+E^{2}\right)^{\frac{3}{2}}}}_{!} \frac{\partial \beta_{n, m-1}}{\partial u} \\
& +\underbrace{\frac{u}{\sqrt{u^{2}+E^{2}}}}_{!} \frac{\partial^{2} \beta_{n, m-1}}{\partial u^{2}} \\
& \frac{\partial^{2} \beta_{n, 0}}{\partial u^{2}}=2 \frac{R}{u^{3}} \beta_{n-1,0}-2 \frac{R}{u^{2}} \frac{\partial \beta_{n-1,0}}{\partial u}+\frac{R}{u} \frac{\partial^{2} \beta_{n-1,0}}{\partial u^{2}} \\
& \frac{\partial^{2} \beta_{0,0}}{\partial u^{2}}=2 \frac{R}{u^{3}}
\end{aligned}
$$

